\title{
Optimization of heat transfer at the surface of parabolic fins by genetic Algorithm
}

\author{
A. Gholami ${ }^{1}$ and H. Mehrjou ${ }^{2 *}$ \\ ${ }^{I}$ Mechanical Engineering, Faculty of Engineering, Islamshahr Branch, Islamic Azad University, Islamshahr, Iran \\ ${ }^{2}$ Islamshahr Branch, Islamic Azad University, Islamshahr, Iran
}

\begin{abstract}
Thermal fins are extended surfaces like longitudinal, radial, and cylindrical fins. They are used for the improvement of heat transfer between an object and fluid. Fins can be applied in many processes of objects cooling such as electrical appliances, many types of engines, transformers, chemical industry, air conditioning, heat exchangers and the industries of energy exchanges. This matter is one of the main results of paying attention to this issue. Therefore, this study examined the optimization of fin heat transfer by using a genetic algorithm and consideration of Nusselt number and hydraulic resistance as the objective function. In this paper, we investigated the optimization of the fin to maximize the heat transfer and also to minimize the hydraulic resistance. The results of this study can be effective in term of technical and economic efficiency in the industry of fin transformer. The stimulation and library method has been used to collect data. The results indicated that if the hydraulic resistance was limited to a specific value, using wavy fins will not improve the device heat.
\end{abstract}

Keywords: Heat transfer optimization; Parabolic fins; Genetic algorithm; Natural convection; Effectiveness

\section{Introduction}

Using of fins is too important when we cannot increase the heat transfer coefficient and the temperature difference between object and fluid (Tong, 2011). Calculation of fin efficiency in heat exchanger engineering is too important because of the evaluation of fin surface performance and determination of the coefficient of heat transfer from experimental data. The aim of optimization is to find the number and shape of fins and their way of placement beside each other in a way that achieve the most rate of heat transfer.

L. C. Ngo presented work on the numerical modeling and optimization of natural convection heat suppression in a solar cavity receiver with plate fins (Ngo et al. 2015). The results of study showed that a significant reduction on the natural convection heat loss can be achieved from cavity receivers by using plate fins. They found that natural convection heat loss is dependent on the receiver, inclinationangle, fins height and thickness. Mosayebi dorcheh investigated an optimum design point for fin geometry and the performance and optimum design analysis of straight convective-radiative fins with internal heat generation of four special different geometries, namely, rectangular, convex, triangular and concave types (Mosayebidorcheh et al. 2015). Results indicated that by increasing the fin thicknesses ratio, maximum heat transfer rate decreases and Copper among the other materials has the most heat transfer rate in a constant volume.

In this paper, we investigated the optimization of the fin to maximize the heat transfer and also to minimize the hydraulic resistance. The modified genetic algorithm was used in order to achieve the optimized coordination of control points and the number of fins. Finally the effect of fin height change over fin length, fin base temperature overfin optimized geometry and the optimum number of fins has been examined. The research objective is to maximize the heat transfer and minimize hydraulic resistance. According to the fact that two objective functions are in opposite of each other, the results of genetic algorithm present a solution for each of the functions to be placed in the equilibrium curve. The results showed that if agenetic algorithm is used without any indicator, all the produced geometries are acceptable and as a result the algorithm convergence rate rises sharply.

\section{Materials and methods}

Consider a conduit consists of the finned plate against a flat plate. The flat plate in thermal view during the moment heating of "q" flux is transmitted to the bottom of the finned plate. Cold flows in duct and fins are equal. Orthogonal sections have two symmetry axes in direct current. 
Heat transfer performance could be studied only in order to share of what has been determined with the bottom of the finned plate. Let's select an orthogonal and compatible system which the axis $\mathrm{x}$ is parallel to the direction of flow and the $y$-axis is perpendicular to the plate when it is parallel to the $z$. Figure 4 indicated the calculation area where "a" is fin height, " $b$ " is the base of the thickfinned, "e" is the distance between the two symmetrical axes, and " $\mathrm{d}$ " is the distance between finned base and flat plate.

Calculation of the range geometry has been showed with e, a, $\mathrm{b}$, and $\mathrm{d}$. And $\mathrm{g}(\mathrm{y})$ is the performance feature finned. We considered "d" as a longitudinal reference and also obtains the following dimensionless variables:

$$
\mathrm{a}=\frac{\mathrm{a}}{\mathrm{d}} ; \beta=\frac{\mathrm{b}}{\mathrm{d}} ; \varepsilon=\frac{\mathrm{e}}{\mathrm{d}} ; \Phi(\eta)=\frac{\mathrm{g}(\mathrm{y})}{\mathrm{d}} ; \eta=\frac{\mathrm{y}}{\mathrm{d}} ;
$$

The structural polynomial for function future $\Phi$ is as follow:

$$
\Phi(\eta)=\sum_{\mathrm{i}=0}^{\mathrm{n}} \psi_{\mathrm{i}} \eta_{\mathrm{i}}
$$

The operation of has been defined through $n+1$ parameters of $\psi_{i}$, or in a case of replacement values of $n+1$ from $\Phi$ in front of the values on $\eta$ axis:

$$
\Phi_{\mathrm{i}}=\sum_{\mathrm{i}=0}^{\mathrm{n}} \psi_{\mathrm{i}} \eta_{\mathrm{i}}
$$

We first change the variables of $\Phi$ which have more calculable nature than $\left(\psi_{\mathrm{i}}\right)$ in . the average of the half fin width is shown as follow:

$$
a=\beta+\frac{a \Phi}{\varepsilon}
$$

Here, 6 is the thickness of the finned and is shown as follow:

$$
\Phi=\sum_{\mathrm{i}=0}^{\mathrm{n}} \frac{\Psi_{\mathrm{i}}\left(\Phi_{0}, \ldots, \Phi_{\mathrm{n}}\right)}{\mathrm{i}+1}
$$

This parameter is the agent of value and the weight of the plate finned.

Accordingly, for optimizing a plate finned, we can increase the Nusselt number and at the sametime, we can reduce the hydraulic resistance or developed entropy. Also, the average thickness can be limited to a certain amount which this limitation for $\sigma$ has been applied in order to reduce the value of finned. For achieving this purpose $\varepsilon$ and $\Phi$ can be reproduced which are carried out through appropriate genetic operation while the value of $\beta$ can be as follow:

$$
\beta=\sigma_{0}-\frac{a}{\varepsilon} \Phi
$$

In this case, $\beta$ can be negative or very small. In this situation,
$\Phi$ has the ability to resize or the combination of parameters can be rejected by designing the zero value for Nusselt number and a limited value for the hydraulic resistance.

In terms of the simulation, only $\mathrm{x}$ component of $\mathrm{u}$ of fluid velocity is non-zero and it can achieve by solving the corresponding component of the momentum equation:

$$
\frac{\partial^{2} u}{\partial y^{2}}+\frac{\partial^{2} u}{\partial x^{2}}=\frac{1}{\mu} \frac{\partial p}{\partial x}
$$

Here, the $\mu$ is dynamic speed and the $\mathrm{p}$ is pressure. The temperature distribution in coolant can be obtained via following equation:

$$
\rho c_{p} u \frac{\partial \mathrm{T}_{\mathrm{c}}}{\partial \mathrm{x}}=\mathrm{k}_{\mathrm{c}}\left[\frac{\partial^{2} \mathrm{~T}_{\mathrm{c}}}{\partial \mathrm{y}^{2}}+\frac{\partial^{2} \mathrm{~T}_{\mathrm{c}}}{\partial \mathrm{z}^{2}}\right]
$$

$\rho$ is density, $\mathrm{cp}$ is specific heat, and $\mathrm{k}_{\mathrm{c}}$ is thermal conductivity of the coolant. The temperature distribution within the finny sheets has been described with the Laplace equation:

$$
\frac{\partial^{2} \mathrm{~T}_{\mathrm{f}}}{\partial \mathrm{y}^{2}}+\frac{\partial^{2} \mathrm{~T}_{\mathrm{f}}}{\partial \mathrm{z}^{2}}=0
$$

The two aforementioned equations should satisfy the following conditions:

- On the contact surface the temperature in the solid is equal to liquid

- Heat flux towards the normalization is equal to the amount of solid and liquid on the contact surface

- Heat flux in normal direction must be zero in the axis of symmetry and on the wall insulation.

- Heat flux should be equal to $\mathrm{q}$ and parallel to the $\mathrm{Y}$ direction on finny at low level

\section{Results and discussions}

The aim of this study is to optimize the heat transfer at the surface of the parabolicfin by using a genetic algorithm. According to the results of previous studies, we can achieve a geometry that assures the maximum value of heat transfer by genetic algorithm. While the shape and geometry itself may be ineffective because it limit the hydraulic resistance and the volume of fins may be increased. Therefore, this study examined the optimization of fin heat transfer by using a genetic algorithm and consideration of Nusselt number and hydraulic resistance as the objective function. The research 
problem is to maximize the heat transfer and minimize hydraulic resistance. According to the fact that two objective functions are in opposite of each other, the results of genetic algorithm present a solution for each of the functions to be placed in the equilibrium curve. A genetic algorithm is a simple but powerful tool. The genetic algorithm considers several points of search space at the same time. Therefore it reduces the chance of converging to a local minimum. In this algorithm, the calculation of the value of each point is



Fig. 1. Webbed ducts

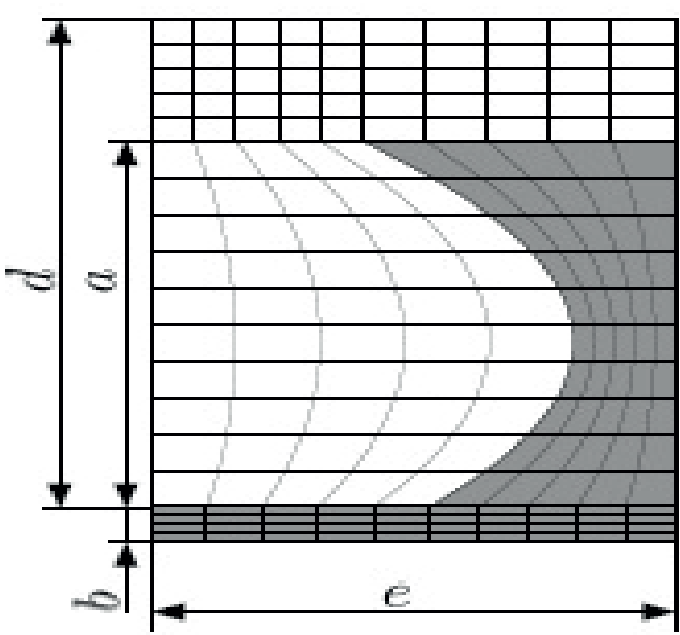

Fig. 2. the geometry range

conducted independently. Spontaneous calculation of several points is applicable to parallel computers. This algorithm does not need much information about the problem because this method in any period by calculation of the value of each variable and production of new collections by using its operators to produce variable collections which create the best value in the problem. By repetition of this action, it will

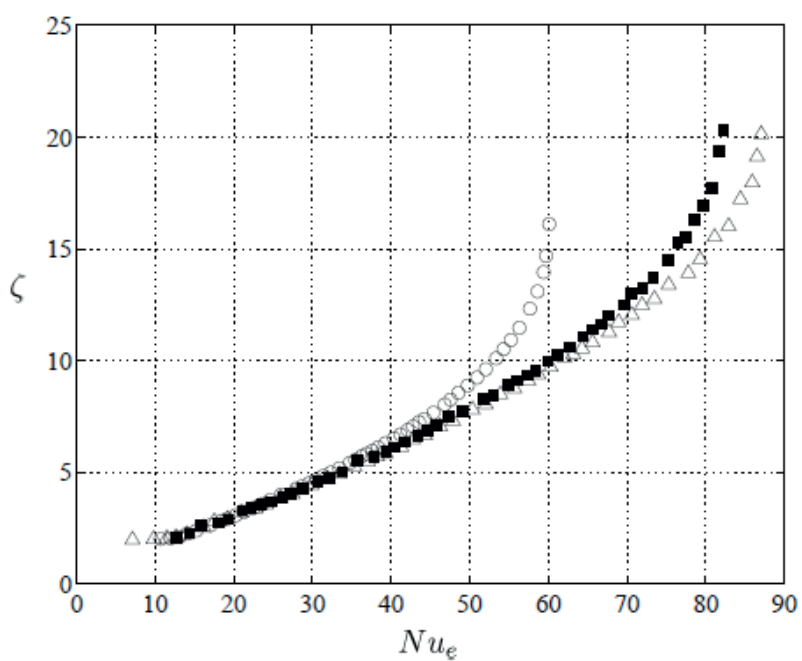

Fig. 3. Pareto front unlimited status: (O) Rectangular fins ( $\square)$ parabolic fin $(\Delta)$ order fin

reach to a set of points. According to the aim of this study, the issue of multi-purpose optimization by the genetic algorithm will be solved in MATLAB software.

In figure 1 we consider a conduit consists of the finned plate against a flat plate, which both are unlimited in the term of longitudinal and transverse. The flat plate in thermal view during the moment heating of "q" flux is transmitted to the
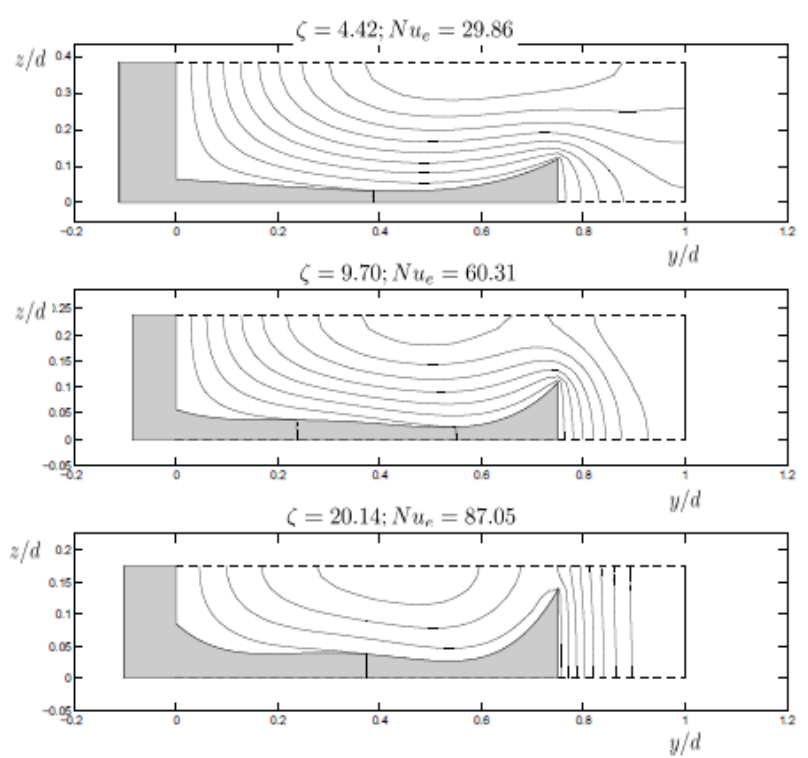

Fig. 4. Temperature distribution in transversal section form $=4$ and $a=0.75$. The curves are drawn in each 10 percent difference between maximum and minimum temperature 
bottom of the finned plate. Cold flows in duct and fins are equal. The calculation of the geometry range has been indicated with e, a, b, and d in Figure 2 where $g(y)$ was the performance feature finned. Orthogonal sections have two symmetry axes in direct current. Since this method uses technical optimization with a high prevalence of persons the simplicity of this model is mandatory. In fact, in order to test cases, 100 individuals have been considered for 100
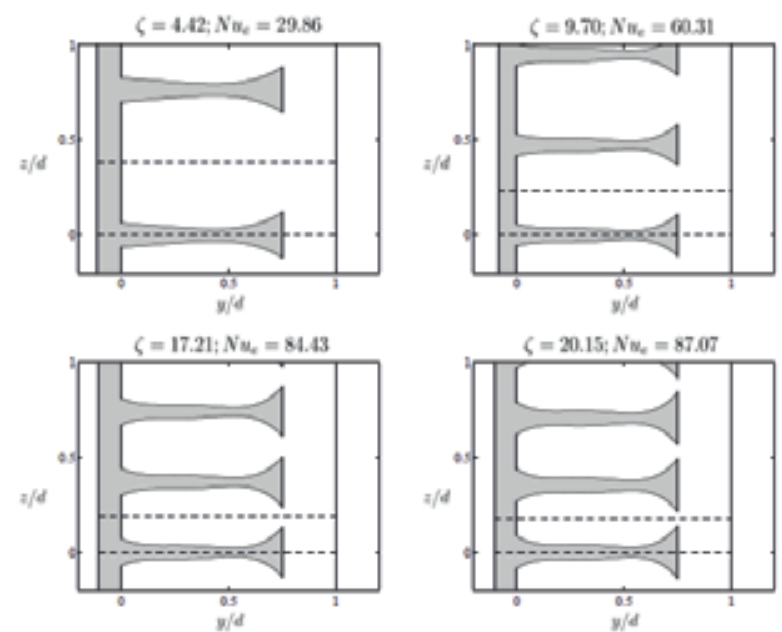

Fig. 5. Optimal geometry for $n=4$

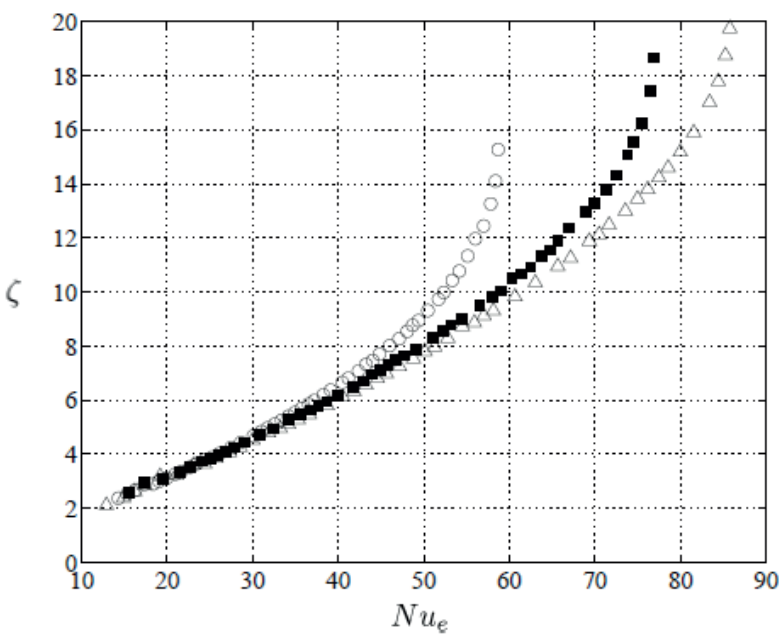

Fig. 6. Pareto front in the condition of the solid unlimited substance $\bar{\sigma}_{0}=0.2(O)$ Rectangular fins ( $\square)$ parabolic fin $(\Delta)$ order fin

generations in order to determine the truth of the Pareto (Figure 3). Therefore, we need 10000 evaluations of cost functions. The use of such solvents is not permitted for such a large number of individuals. Accordingly, we should consider the following:

- The fluid currents of sheet are thin and incompressible;

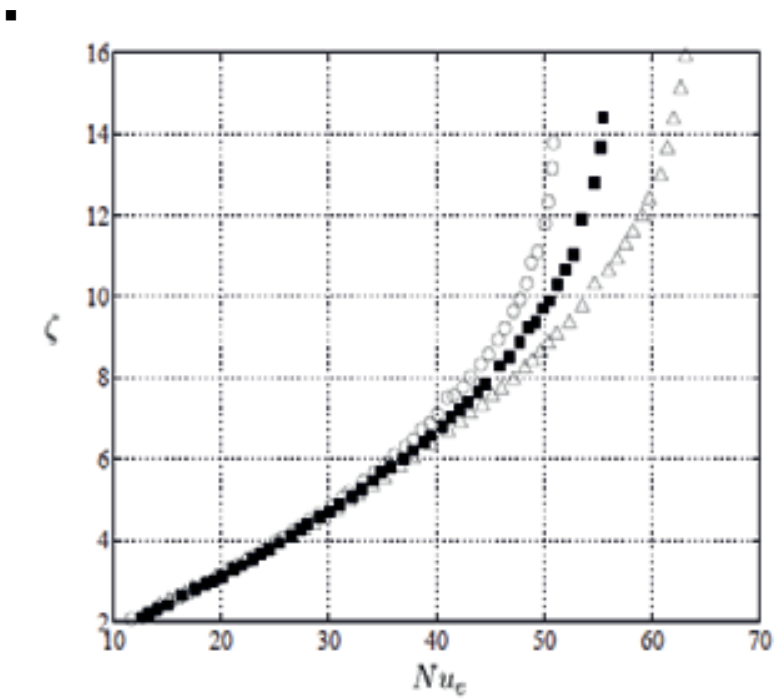

Fig. 7. Pareto front in the condition of solid limited substance $\bar{\sigma}_{0} \quad 0.1=(0)$ Rectangular fins (匹) parabolic fin $(\Delta)$ order fin


Fig. 8. Optimal geometry for $n=4$ with a solid limit substance $\bar{\sigma}_{0}=0.1$ 
- Speed and temperature reached to extremes;

- True convection is negligible due to convection force;

- Viscous dissipation is negligible.

Figure 4 illustrate the temperature distribution in transversal section form $=4$ and $a=0.75$. The curves are drawn in each 10 percent difference between maximum and minimum temperature. Figure 6 indicated two different amounts of the finny which the (the average thickness of the finny) sizes are 0.1 and 0.2. The fluid flow includes thin and incompressible sheets. In fact, in this simulation the following statements are true:

- The fluid flow is laminar and Incompressible.

- The system is sustainable.

- Speed and temperature are developed completely.

- The fluid properties is uniform, and liquid is independent.

- The negligible natural convection is related to the forced convection.

- Viscous dissipation is negligible.

The global thermal heat transfer coefficient can be defined as follow:

$$
h=\frac{q^{\prime \prime}}{T_{\max }-T_{b}}
$$

$\mathrm{T}_{\max }$ is the maximum temperature on the surface, $\mathrm{q}$ " is imposed temperature, and $\mathrm{T}_{\mathrm{b}}$ is fluid mass temperature.

Nusselt number equivalent is defined as follows:

$$
\mathrm{Nu}_{\mathrm{c}}=\frac{2 \mathrm{hd}}{\mathrm{k}_{\mathrm{c}}}
$$

Also the normal hydraulic resistance is defined as follow:

$$
\zeta=\frac{-\mathrm{dp} / \mathrm{dx}}{\mathrm{w}_{\mathrm{t} / \mathrm{e}}} / \frac{12 \mu}{\mathrm{d}^{3}}
$$

Here, only three values of $n$ polynomial have been studied which include:

$$
\mathrm{n}=1,2,4
$$

The approach of optimization for optimizing heat transfer by dissipater, the cooled heat is applied by the light flow. Specifically, the optimal geometry of wavy fins related to the Polynomial functions and parameter compounds that have been studied with the aim of maximizing the heat transfer and hydraulic resistance. At following, you will read the output of the simulation (Figure 5-10).

\section{Conclusion}

Technical and economic studies of thermal power plants and simulating the outcomes lead to the presentation of the best courses of actions in selecting parabolic fins. More optimization and evolution of these systems can help the heat transfer management. In general, the replacement of solar energy with fossil fuels is too expensive. Therefore the results of this study can be effective in term of technical and economic efficiency in the industry of fin transformer. The results of a group of solutions are on the curve which is known as Pareto front.The value of hydraulic of resistance is less than the calculated value in heat transformer profile. In addition, we conclude that if the hydraulic resistance was limited to a specific value, using wavy fin will not improve the heat transfer of device.

\section{References}

Mosayebidorcheh S, Hatami M, Mosayebidorcheh T and Ganji DD (2015), Optimization analysis of convective-radiative longitudinal fins with temperature-dependent properties and different section shapes and materials, Energy Conversion and Management 106: 1286-1294. 
Ngo LC, Bello-Ochende T and Meyer JP (2015), Numerical modelling and optimisation of natural convection heat loss suppression in a solar cavity receiver with plate fins, Renewable Energy 74: 95-105.
Tong XC (2011), Advanced materials for thermal management of electronic packaging, Springer Science \& Business Media.

Received: 27 February 2017; Revised: 30 April 2017; Accepted: 29 May 2017. 